\title{
Sensitive sensors based on bilayer organic field-effect transistors for detecting lithium-ion battery electrolyte leakage
}

\author{
Shiqi Zhang ${ }^{1}$, Yang $\mathrm{Lu}^{1}$, $\mathrm{Li} \mathrm{Li}^{1}$, Xin Wang ${ }^{1}$, Dapeng Liu ${ }^{1}$, Junyao Zhang ${ }^{1}$, Shilei Dai ${ }^{1}$, Dandan Hao ${ }^{1}$, \\ Ben Yang ${ }^{1}$, Quan Sun ${ }^{2}$, Yunhui Huang ${ }^{2}$, Lai $\mathrm{Wei}^{3}$ and Jia Huang ${ }^{1,4^{*}}$
}

\begin{abstract}
The leakage of flammable and explosive lithiumion battery (LIB) electrolytes can be one of the early symptoms of battery malfunction and can even lead to spontaneous battery combustion or electric car explosion. Therefore, it is necessary to find a rapid and simple method to monitor any leakage of LIB electrolytes. However, LIB electrolytes are generally composed of volatile and redox neutral carbonate solvents. Trace amounts of electrolyte leakage are difficult to detect effectively and rapidly by existing compact sensors. Here, for the first time, we propose a strategy that cooperatively combines the sensitivity of organic field-effect transistors (OFETs) and the selectivity of biurea receptors to detect LIB electrolyte leakage. The fabricated sensors show much higher sensitivity than the pristine sensor without receptors, and the detection limit of the sensor toward diethyl carbonate was $1.4 \mathrm{ppm}$. Trace amounts of LIB electrolyte leakage could be detected effectively in seconds, with $200 \mathrm{~nL}$ electrolyte leakage leading to a $3 \%$ response. We also demonstrate the real-time detection of LIB electrolyte leakage by our OFET sensors. The excellent performance of the receptorcoated OFET sensor makes it a good candidate for LIB safety monitoring and provides a promising platform for the development of sensing technologies.
\end{abstract}

Keywords: organic field-effect transistors, chemical sensor, receptor, lithium-ion batteries, electrolyte leakage

\section{INTRODUCTION}

With continuous developments in science and technology, human demand for electric power storage continues to grow. Lithium-ion batteries (LIBs) are one of the most important electric storage systems due to their advantages of high energy density, good cycle performance and environmental friendliness [1]. LIBs have been widely used in people's daily lives from portable electronics to electric cars. However, if LIBs are misused or encounter abnormal environmental conditions, accidental scenarios may be triggered. In recent years, occasional incidents such as mobile phone explosions and electric car selfignition have often caused severely debilitating injuries and even fatal tragedies [2], which have led to the withdrawal of products, loss of market share and even a ban on LIBs under certain circumstances. LIB failure can be accompanied by the leakage of flammable and explosive organic solvent-based electrolytes, and the released electrolyte vapor can cause the situation to become uncontrollable [3]. Thus, it is important to monitor any leakage of LIB electrolytes, not only in LIB-powered phones and electric cars for safety purposes but also in the LIB manufacturing process for quality control [4]. Several strategies have been exploited to detect LIB electrolyte leakage in laboratories, such as gas chromatography/mass spectrometry (GC/MS) [5] and nuclear magnetic resonance (NMR) spectrometry [6]. However, these kinds of analysis strategies tend to require expensive equipment and long operating time, which are not applicable in real-time electrolyte detection in daily life. Hence, the development of high-performance, compact and portable chemical sensors targeting LIB electrolytes is essential. Basically, LIB electrolytes are mainly composed of redox neutral carbonates such as diethyl carbonate (DEC), dimethyl carbonate (DMC), methyl ethyl carbonate (EMC), ethylene carbonate (EC), and propylene carbonate (PC), with small amounts of other chemicals [3]. These redox neutral carbonates are difficult to detect using existing sensors. Studies in this research field are essential but rare. Therefore, there is an urgent need to develop a sensitive, facile and low-cost electrical sensor to monitor LIB electrolyte leakage.

Sensors based on organic field-effect transistors (OFETs) show great potential due to their high sensitivity, simple manufacturing process, low cost, flexibility, and room temperature operation [7-9]. Various OFET sensors, including chemical sensors, biosensors, humidity sensors, pressure sensors, temperature sensors, and integrated sensor arrays with advanced functionalities, have been extensively studied and reported [1013]. Compared with conventional two-terminal devices, the electrical output signals of sensors based on OFETs can be significantly amplified and tuned by modulating their gate voltage [14-16]. However, typical OFET-based sensors show limited sensitivity to redox neutral carbonate chemicals due to the weak interactions between analytes and organic semiconductor (OSC) molecules. To develop high-performance chemical sensors, relevant strategies commonly adopted include material design [17-19], device configuration [20,21], active-layer thickness

\footnotetext{
${ }^{1}$ Interdisciplinary Materials Research Center, School of Materials Science and Engineering, Frontiers Science Center for Intelligent Autonomous Systems, Tongji University, Shanghai 201804, China

${ }^{2}$ Institute of New Energy for Vehicles, School of Materials Science and Engineering, Tongji University, Shanghai 201804, China

${ }^{3}$ Hangzhou Yanqu Information Technology Co., Ltd., Hangzhou 310003, China

${ }^{4}$ Translational Research Institute of Brain and Brain-Like Intelligence, Shanghai Fourth People's Hospital, Tongji University, Shanghai 200434, China

* Corresponding author (email: huangjia@tongji.edu.cn)
} 
modulation [22-25], functional receptor implantation [26-29], and device geometry optimization [30-33]. Among them, the incorporation of functional recognition units into the surface of OSC films can endow OFET chemical sensors with specific recognition capabilities and ideal sensing capacity, thereby selectively distinguishing target analytes [34,35].

Here, for the first time, we reported LIB electrolyte sensors based on biurea-decorated OFETs. The sensors showed much higher sensitivity than the pristine sensor without receptors, and trace amounts of LIB electrolyte leakage could be detected effectively within seconds. When only $200 \mathrm{~nL}$ of LIB electrolyte was released, the device already showed a 3\% current decrease. Moreover, real-time LIB pack leakage was simulated, and satisfactory early warning signals were obtained, which demonstrates that our sensors are promising candidates for LIB safety monitors. The current high-performance OFET device based on the receptor layer should have important implications for the development of new sensing technologies.

\section{EXPERIMENTAL SECTION}

\section{Materials}

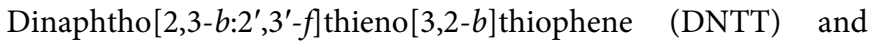
biurea were purchased from TCI. The LIB electrolyte LBC305-1 was purchased from Shenzhen Capchem Technology. This electrolyte is a colorless liquid with a microfragrance. The electrolyte salt is $\mathrm{LiPF}_{6}$. The organic solvents are DEC, EC and EMC (1:1:1 in volume). The density at $25^{\circ} \mathrm{C}$ is approximately $1.23 \pm 0.03 \mathrm{~g} \mathrm{~mL}^{-1}$. The boiling point is approximately $90-$ $248^{\circ} \mathrm{C}$. The melting point is less than $-20^{\circ} \mathrm{C}$. The conductivity at $25^{\circ} \mathrm{C}$ is approximately $10.4 \pm 0.5 \mathrm{mS} \mathrm{cm}^{-1}$. The vapor pressure at $20^{\circ} \mathrm{C}$ is approximately $8 \mathrm{mmHg}$.

\section{Fabrication of OFET devices}

Heavily n-type doped Si wafers with $300 \mathrm{~nm}$ thermally grown $\mathrm{SiO}_{2}$ layer were used as substrates. They were first cleaned by sonication in acetone and isopropanol alternately for $1 \mathrm{~h}$, rinsed with deionized water and ethanol, and dried by nitrogen flow. The wafers were immersed in n-octadecyltrimethoxysilane (OTS) toluene solution $(1.2 v / v \%)$ at room temperature for $3 \mathrm{~h}$, followed by washing with toluene and ethanol. Then, 10, 30, and $50 \mathrm{~nm}$ DNTT semiconductor films were thermally evaporated at a rate of $0.1 \mathrm{~A} \mathrm{~s}^{-1}$ and a pressure of $5 \times 10^{-4} \mathrm{~Pa}$. Biurea of $5 \mathrm{~nm}$ was also thermally deposited by vacuum thermal evaporation. Gold source-drain electrodes $(40 \mathrm{~nm})$ were thermally evaporated through a shadow mask to form top-contact OFET devices. The channel length $(L)$ and channel width $(W)$ were 100 and $1000 \mu \mathrm{m}$, respectively.

\section{Device characterization and measurement}

All electrical performance and sensing tests of OFETs were measured by using a Keithley 4200 (Keithley Instrument) semiconductor characterization system connected to a homemade vapor testing chamber. The surface morphology and thickness of DNTT films for the gas sensors were observed by atomic force microscopy (AFM, SEIKO SPA-300HV). ${ }^{1} \mathrm{H}$ NMR spectra were recorded using a Bruker AV $400 \mathrm{MHz}$ spectrometer at room temperature.

\section{Sensing experiments}

The sensing performance of the fabricated devices was mon- itored in a homemade test chamber at room temperature. An electrical feed-through and gas inlet and outlet were installed in the chamber. After the device was placed in air for a period of time, DEC was injected into the chamber. Then the electrical characteristics and response and recovery curves of the devices were recorded.

\section{Density functional theory (DFT) calculations}

DFT calculations were performed using the Gaussian 16 package (M062X-D3/def2tzvp/M062X-D3/def2svp). To be consistent with the experiment, a solvation model based on density (SMD) of water was used in the calculations. The independent gradient model (IGM) method was applied to study the noncovalent interactions between molecules. The IGM analysis was conducted with Multiwfn software. To study electron transfer before and after the combination, electron density difference analysis was carried out. The IGM interaction regions and color mapped isosurface graphs were obtained via the variational mode decomposition (VMD) visualization program.

\section{RESULTS AND DISCUSSION}

OFET-based sensors with biurea as receptors were fabricated with a bottom-gate and top-contact configuration. Heavily ntype doped silicon wafers with $300 \mathrm{~nm} \mathrm{SiO} 2$ were used as substrates. DNTT was selected as the active semiconductor layer because of its known relatively high stability and mobility. OSC films of DNTT were then thermally evaporated onto OTStreated $\mathrm{SiO}_{2} / \mathrm{Si}$ substrates. Source and drain electrodes were formed by evaporating gold through shadow masks. The receptor layer of biurea was thermally evaporated at the top of the channel layer. The deposited biurea film thickness was optimized to be $5 \mathrm{~nm}$. Biurea, as the receptor layer, can be thermally deposited instead of solution preparation so that the morphology can be controlled. The morphology and performance of the semiconductor layer will not be destroyed and greatly affected. Further details on the fabrication of DNTT OFET-based sensors are described in the EXPERIMENTAL SECTION. The molecular structure of DNTT and biurea, the device structure, and the schematic of the experimental apparatus are illustrated in Fig. 1. The device characteristics based on the bare and surface-modified DNTT films were measured and compared. Typical transfer curves and output characteristics are shown in Fig. 2a, b, respectively. Regarding the bias of the transfer characteristics, both devices exhibit typical p-type OFET characteristics, showing clear linear and saturation regimes. The DNTT OFET without biurea has an average field-effect mobility $\left(\mu_{\mathrm{FET}}\right)$ of $1.24 \mathrm{~cm}^{2} \mathrm{~V}^{-1} \mathrm{~s}^{-1}$, with an on/off current ratio $\left(I_{\mathrm{on}} / I_{\mathrm{off}}\right)$ of more than $10^{7}$. After functionalization with biurea, the device exhibits an average $\mu_{\mathrm{FET}}$ of $0.2 \mathrm{~cm}^{2} \mathrm{~V}^{-1} \mathrm{~s}^{-1}$ and an $I_{\mathrm{on}} / I_{\mathrm{off}}$ of higher than $4 \times 10^{4}$. The mobility decreases after functionalization with receptors. The semiconductor DNTT is a p-type semiconductor with holes acting as charge carriers. Biurea is an electron donor molecule. After functionalization with biurea, the electron donating effect of biurea reduces the output current. Fig. 2b shows the $I_{\mathrm{D}}-V_{\mathrm{D}}$ curves of DNTT OFETs functionalized with biurea. The device shows obvious field-effect behaviors for achieving signal amplification while sensing analytes.

The surface morphological characteristics of DNTT films with and without the receptor layer were investigated by AFM analysis. Fig. 2c and Fig. S1 show AFM topography images of DNTT films without biurea. The 10 -nm-thick DNTT film exhibits 

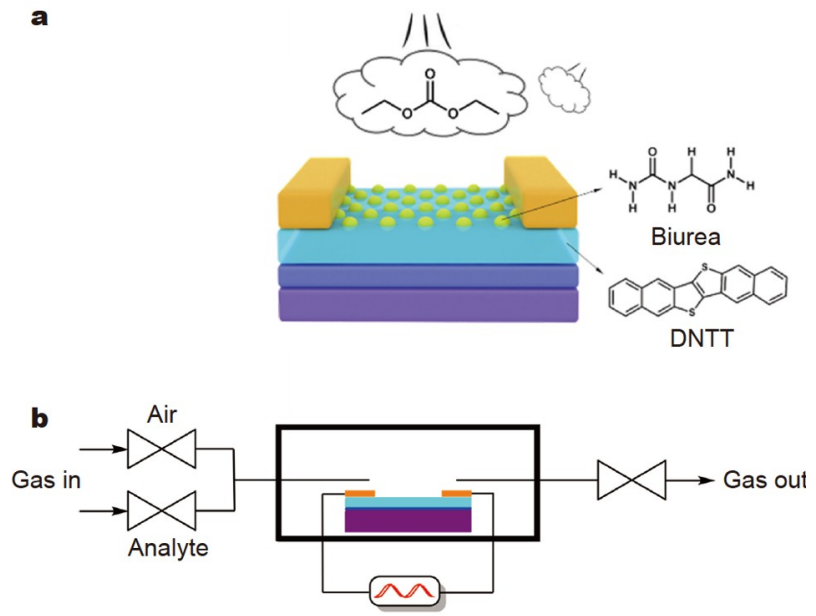

Figure 1 (a) Device configuration of the top-contact OFET-based sensors and the molecular structures of the sensing vapor, DEC, a receptor, biurea, and the sensing layer, DNTT. (b) Schematic of the experimental apparatus.

polycrystalline grains with a root-mean-square (RMS) roughness of $0.47 \mathrm{~nm}$. As seen in Fig. 2d, a uniform biurea film was achieved on top of the DNTT layer. The cross-sectional AFM analysis on a control sample (silicon wafer deposited with biurea) reveals that the thickness of the receptor layer is $5 \mathrm{~nm}$ (Fig. S2). The thin and uniform biurea film provides a decent receptor layer for analyte adsorption (Fig. 2e and Fig. S3). The thin films of DNTT, biurea and DNTT with biurea were characterized by X-ray photoelectron spectroscopy (XPS) (Fig. S4).

Owing to their electrochemical stability, ion solvation properties and abilities to form protective layers, electrolytes based on carbonate solvents such as DMC, DEC or EMC, etc., are applied in most commercial LIBs. Therefore, to simplify the preliminary exploration, DEC was chosen as the target subject to evaluate the sensor's performance. The purpose of this device is to identify the leakage of LIB electrolytes. When testing the electrolyte, all kinds of organic volatiles in the electrolyte will affect the performance of the device, and the components of the electrolyte are complicated. As long as the device can respond quickly when a trace amount of electrolyte leaks, the goal has been achieved. A homemade sensor testing chamber attached to a Keithley 4200 semiconductor characterization system was prepared for the detection of DEC vapors, as shown in Fig. 1b. Sensing experiments were carried out under environmental conditions. To determine the optimal thickness of DNTT films, the sensing performances of devices with different OSC film thicknesses (10, 30 and $50 \mathrm{~nm}$ ) toward $600 \mathrm{ppm}$ DEC vapors were compared. Fig. S5a, b plot the normalized source-drain current $\left(I_{\mathrm{D}} / I_{0}\right)$ response of the three devices at a fixed gate voltage $V_{\mathrm{G}}=-40 \mathrm{~V}$ and $V_{\mathrm{D}}=-10 \mathrm{~V}$ as a function of time. The $I_{\mathrm{D}}$ of the three devices all decreased when exposed to DEC vapors. The higher sensitivity of the sensor with a thinner DNTT film $(10 \mathrm{~nm})$ could be clearly observed due to the easier contact between DEC molecules and the conduction channel layer around the bottom of the semiconductor layer, which showed minimal diffusion barriers in these devices. As the film thickness decreased further, the device performance and long-term stability of the sensor decreased significantly. Thus, the OFET device with a $10-\mathrm{nm}$ thick DNTT film was chosen to study the sensing behaviors upon exposure to different concentrations of DEC vapors. To investigate the function of the receptor layer, we monitored changes in the $I_{\mathrm{D}}$ of the sensors with and without biurea upon exposure to $600 \mathrm{ppm}$ DEC. Fig. S5c shows the comparison of responses between the DNTT OFET devices with and without biurea. The DNTT OFET device with biurea exhibited a much more significant and rapid response $(62 \%)$ than the pristine device without a receptor layer (28\%), indicating that introduction of the receptor layer of biurea on the device surface could significantly enhance the sensor's sensitivity to DEC vapors. Then, we fabricated and characterized biurea receptors with three different thicknesses $(1,5$ and $10 \mathrm{~nm})$. Their morphology and thickness maps are shown in Fig. S2. We also compared the responses of OFET devices loaded with three
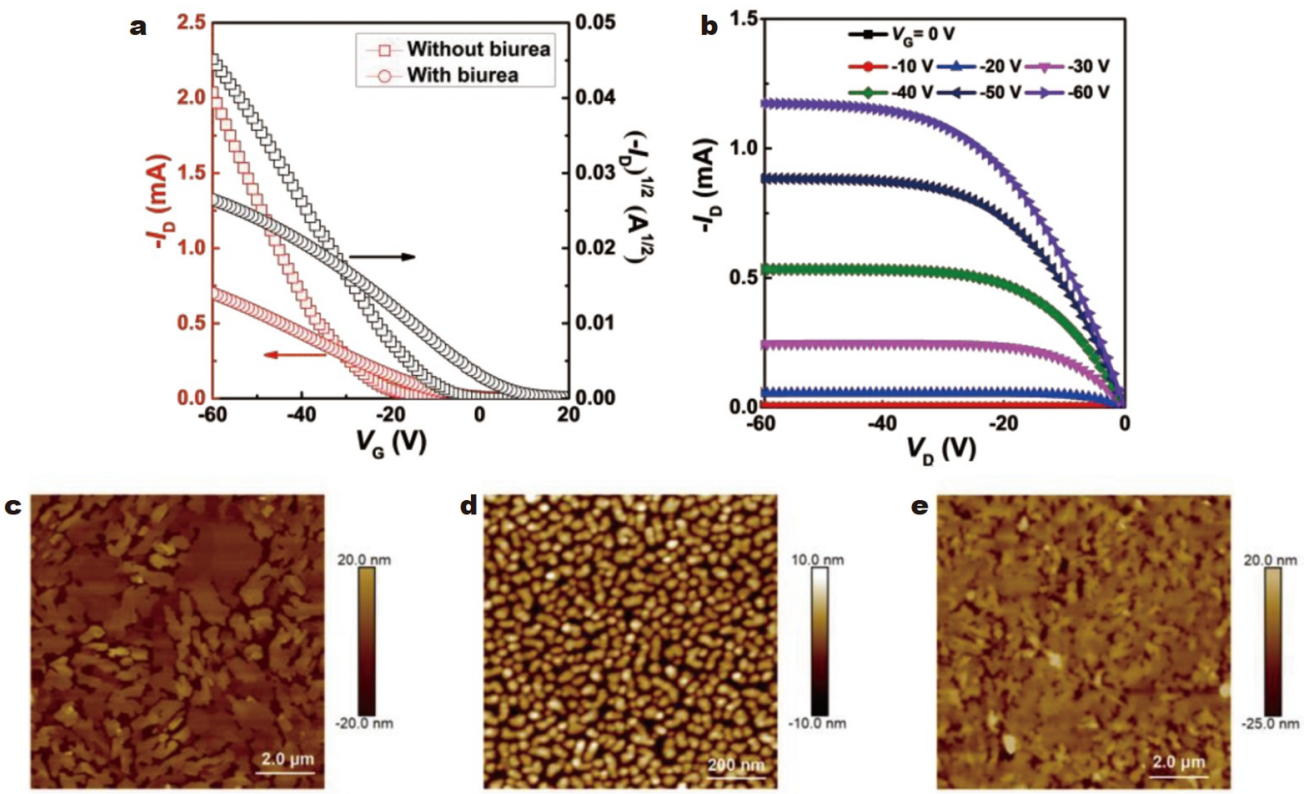

Figure 2 (a, b) Electrical characteristics of DNTT OFETs with and without biurea. (a) Transfer characteristics for DNTT OFETs in p-chanel operation mode. (b) Output characteristics for DNTT OFETs with biurea. AFM imags of (c) $10 \mathrm{~nm}$ DNTT, (d) $5 \mathrm{~nm}$ biurea, and (e) $10 \mathrm{~nm}$ DNTT with $5 \mathrm{~nm}$ biurea. 
different thicknesses of biurea receptors to $600 \mathrm{ppm}$ DEC (Fig. S5d). We found that when the thickness of the loaded biurea receptor was $5 \mathrm{~nm}$, the response was the best. When the receptor layer is too thin, it cannot attract enough target molecules; when the receptor layer is too thick, it will block the contact path between the target and the semiconductor layer and affect the electrical properties of the semiconductor layer.

We speculated that the dipole interaction between the DNTT and the DEC vapor, which in turn affected the hole transport of the p-type semiconductor, was one of the main sources of device response. However, dipole interactions were still too weak to adsorb a sufficient number of DEC molecules on the surface of the pristine semiconductor film to generate a strong response. From the perspective of the molecular structure of DEC, there is a carbonyl functional group. We are looking for a molecule with $\mathrm{N}, \mathrm{O}$ or $\mathrm{F}$ atoms, and these molecules are rich in $\mathrm{H}$ atoms. A hydrogen bond is very likely to form with the carbonyl group on DEC so that the two molecules can attract each other to form a strong interaction. Among them, biurea is a good choice. There are a large number of hydrogen atoms on the amino and imino groups of biurea. This conjecture was confirmed in the experimental section. After the deposition of 5-nm-thick biurea on the surface of the DNTT film, the response of the device was greatly improved (increased from $28 \%$ to $62 \%$ to $600 \mathrm{ppm} \mathrm{DEC).} \mathrm{The}$ fabricated sensors showed much higher sensitivity than the pristine sensor without receptors. The favorable interactions between biurea and DEC were confirmed by theoretical calculations and chemical characterizations.

To explore the sensing mechanism and further understand the favorable interactions between biurea and DEC, theoretical calculations were carried out. The binding structure of biurea and DEC was optimized at the level of M062X-D3/def2tzvp/
M062X-D3/def2svp in the Gaussian 16 software [36]. The optimized conformations of biurea (Fig. 3a), DEC (Fig. 3b) and the combination of biurea and DEC (Fig. 3c) were described. The binding energy between biurea and DEC was evaluated, and the resulting binding energy of $-33.05 \mathrm{~kJ} \mathrm{~mol}^{-1}$ was within the range of conventional hydrogen bonds [37]. The distances from the $\mathrm{H}$ atom of the imino and amino groups of biurea to the $\mathrm{O}$ atom of the carbonyl group of DEC are 2.43 and $2.15 \AA$ in the optimized adduct, respectively. The data are within the range of conventional hydrogen bond distances, suggesting that our models and calculation method are reasonable. Additionally, IGM analysis [38] was carried out to reveal the noncovalent interactions between biurea and DEC. As shown in Fig. $3 d-f$, the weak interaction between molecules can be indicated by the IGM isosurface color map and the volume of the interacting regions between biurea and DEC. In the IGM analysis, blue represents stronger and more stable interactions (such as hydrogen bonds), green represents weak interactions (such as van der Waals-type interactions), and red represents steric resistance interactions. The analysis clearly shows that the interactions between biurea and DEC are comprised of hydrogen bonding interactions $(\mathrm{N}-\mathrm{H} \cdots \mathrm{O}$, as shown in blue) and van der Waals interactions (shown in green). The imino and amino groups actually correspond to different modes of action. The imino group in the middle forms the hydrogen bond. To more accurately and deeply study the molecular configuration, electron density difference analysis was employed to study the electron transfer before and after the combination of biurea and DEC (Fig. S6). The imino group in the middle of biurea has a strong interaction with the DEC molecule. The blue bubble area represents the area where electron density decreases, and the green bubble area represents the area where electron density
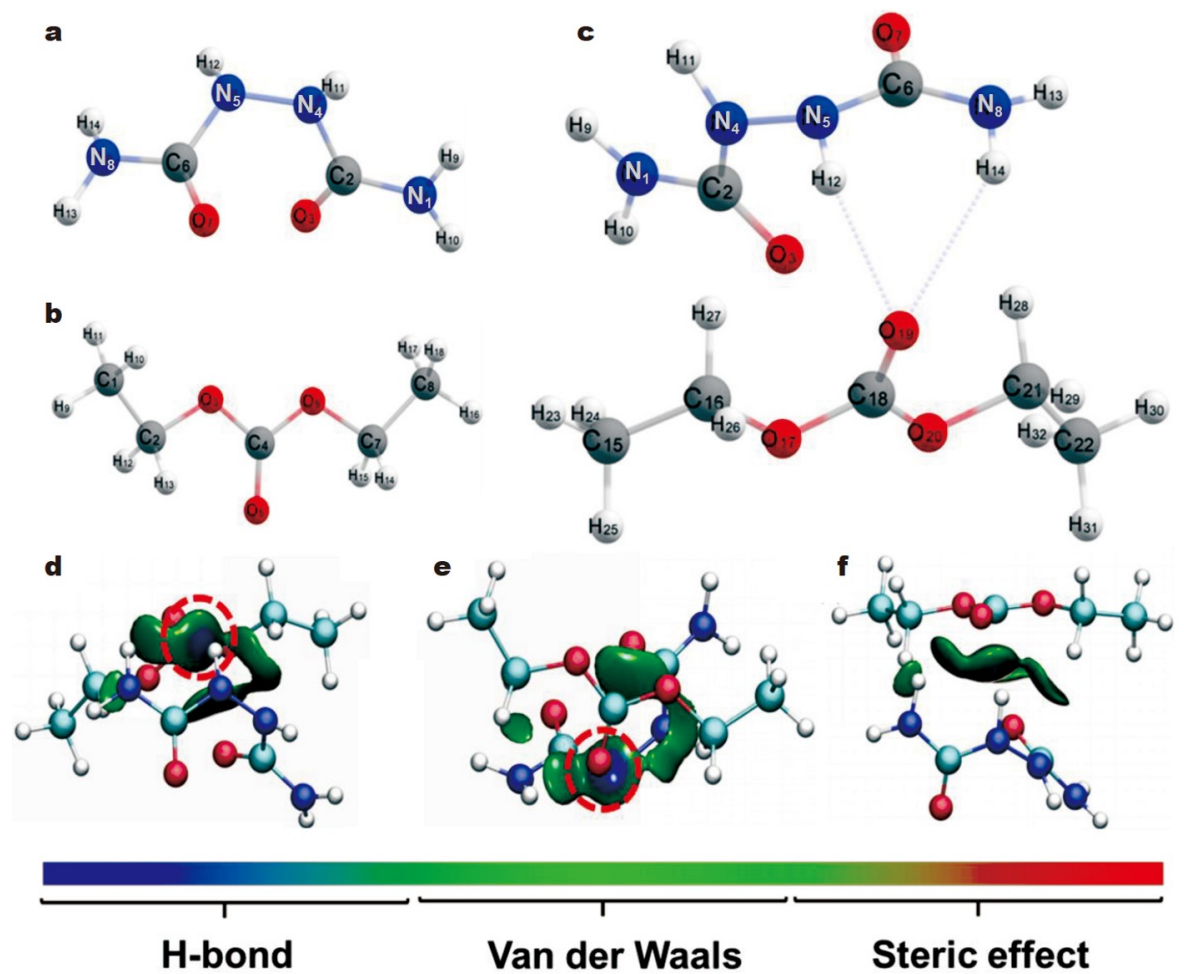

Van der Waals

Steric effect

Figure 3 DFT optimized structures of (a) biurea, (b) DEC and (c) combination of biurea and DEC. (d, e, f) Non-covalent interactions between biurea and DEC using IGM. 
increases. These estimates confirm hydrogen bonding in the interaction between biurea and DEC. The optimized conformations of the combination between biurea and DMC, EMC, EC and PC are described in Fig. S7. Details of the computational study can be seen in Table S1. The DNTT OFET sensor with biurea can also show good sensing performance to DMC vapors (Fig. S8). Therefore, we speculated that the strong hydrogen bond between biurea and DEC contributed to the excellent sensing performance of the device targeting redox neutral carbonates. Computational studies also demonstrate that our devices can have a wide detection range and high sensitivity for various redox neutral carbonates in LIB electrolytes. Under the action of a large number of hydrogen bonds, more DEC molecules could be attracted. Strong electric fields can be induced by the adsorption and diffusion of DEC molecules into the DNTT layer. Then, some of the mobile charge carriers in the semiconductor layer can be either trapped or effectively retarded by the DEC molecule dipoles. To further study the effect of biurea receptor layers on $\mathrm{DEC}$, we investigated the binding properties of biurea to DEC in dimethyl sulfoxide (DMSO) $-\mathrm{d}_{6}$ solution using proton ${ }^{1} \mathrm{H}$ NMR spectroscopy. The ${ }^{1} \mathrm{H}$ NMR spectra of DEC and a 1:1 mixture of DEC and biurea are shown in Fig. S9. The ${ }^{1} \mathrm{H}$ NMR spectra of the raw DEC and biurea are shown in Fig. S10. In the DEC molecule, carbonyl groups are electron withdrawing to the methylene and methyl groups. However, after the introduction of biurea, the imino group of biurea forms hydrogen bonds with the carbonyl group in DEC, and the carbonyl group strengthens the electron withdrawing effect of the imino group on biurea, which can affect the methylene and methyl groups in the DEC molecule. The signals of methylene protons and methyl protons next to the carbonyl group of DEC are remarkably shifted upfield compared with those of free DEC.

To further investigate the chemical sensing performance of the device for DEC vapors, the $I_{\mathrm{D}}$ changes of the devices at a fixed $V_{\mathrm{D}}$ and $V_{\mathrm{G}}$ were monitored at different concentrations of DEC vapors (Fig. 4a). The DNTT OFET sensor with biurea exhibited
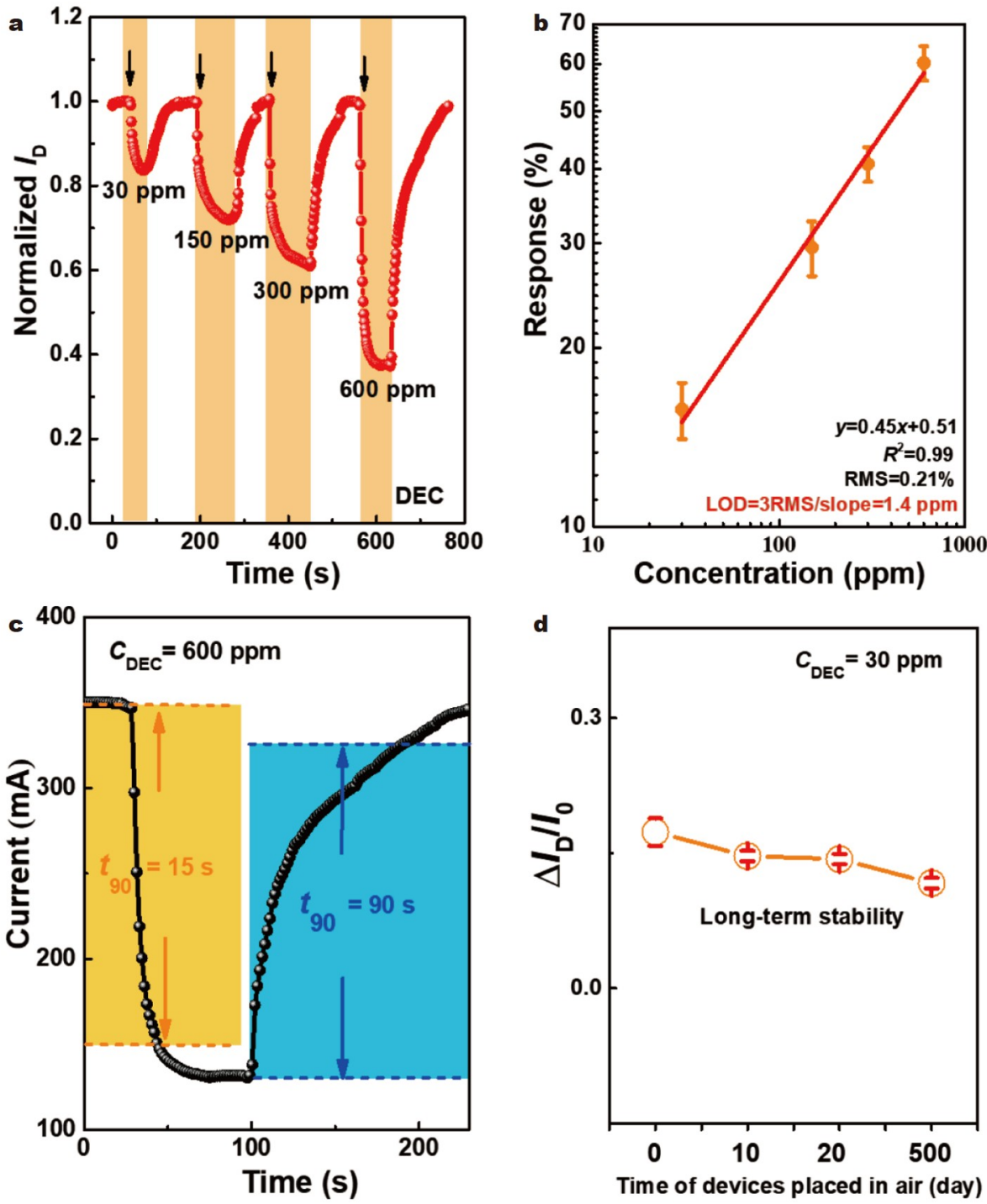

Figure 4 (a) The time-dependent responses of the DNTT OFET with biurea receptor upon exposure to DEC vapors at concentrations ranging from 30 to $600 \mathrm{ppm}$. (b) Linear fit of time-dependent responses of the DNTT OFET with biurea receptor upon exposure to DEC vapors at concentrations ranging from 30 to $600 \mathrm{ppm}$. (c) Response-recovery time curves of the DNTT OFET with biurea receptor to 600 ppm DEC vapor. (d) The long-term stability of the DNTT OFET with biurea receptor upon exposure to $30 \mathrm{ppm}$ DEC vapor. 
$16 \%$ current decay rapidly when exposed to $30 \mathrm{ppm}$ DEC vapor. Rapid current decreases of $28 \%, 39 \%$ and $62 \%$ were observed when the sensor was exposed to doses of 150,300, and $600 \mathrm{ppm}$ DEC vapors, respectively. Linear fit of time-dependent responses of the DNTT OFET with biurea receptor upon exposure to DEC vapors at concentrations ranging from 30 to $600 \mathrm{ppm}$ was plotted with error bars and is illustrated in Fig. $4 \mathrm{~b}$. The sensor's limit of detection (LOD) was $1.4 \mathrm{ppm}$. Fig. 4c shows the response time (15s) and recovery time $(90 \mathrm{~s})$ of the DNTT OFET sensor upon exposure to $600 \mathrm{ppm}$ DEC vapor and dry air, respectively, which are sufficient for real-time detection. The OFET characteristics after exposure to DEC are shown in Fig. S11. Figs S12 and S13 show nonnormalized responses of DNTT OFET sensors to 30 to $600 \mathrm{ppm}$ DEC. As shown in Fig. S13, $V_{\mathrm{G}}$ and $V_{\mathrm{D}}$ are fixed at -10 and $-10 \mathrm{~V}$, respectively. The low operating voltage further ensures the possibility of future practical applications of our devices. In practical applications, LIB electrolyte leakage monitoring sensors tend to be sealed in a closed package beside the battery, which limits the presence of potential interference analytes. Therefore, the high sensitivity and fast response speed to electrolyte leakage rather than selectivity are the primary requirements of the device. The selectivity of the sensor was still investigated, and the crosssensitivities of fabricated sensors toward common organic vapors (hexane, toluene, dichloromethane $\left(\mathrm{CH}_{2} \mathrm{Cl}_{2}\right)$, chloroform $\left(\mathrm{CHCl}_{3}\right)$, methanol $\left(\mathrm{CH}_{3} \mathrm{OH}\right)$, and DEC) are shown in Fig. S14. The DNTT OFET sensors functionalized with biurea showed a stronger response to DEC than the other organic vapors tested, which should be enough for this particular application since the presence of potential interference analytes is limited by the sealing of the batteries in the closed container. In the later stage, the selectivity of the sensor can be further increased by incorporation of different types of receptors. Fig. S15 illustrates that six DNTT OFET sensors also showed high performance uniformity towards $30 \mathrm{ppm}$ DEC. Moreover, the device exhibited decent long-term stability and reproducibility over a 20 -day test. The device still showed high sensitivity to $30 \mathrm{ppm}$ DEC, even after 500 days in air (Fig. 4d). Our devices were relatively stable before and after exposure to DEC over the 4000-s test. Under different humidity and temperature environments, the current value did not change significantly, demonstrating the decent stability of the devices (Fig. S16). The DNTT OFET with the biurea device detects the same concentration of DEC vapor in various atmospheres at different temperatures and humidity. The response under the two atmospheres was not significantly different (Fig. S17). The responsivity shown in the figure is sufficient for LIB leak detection to meet practical requirements.

Under the premise of ensuring that the sensor has sufficient sensitivity to DEC vapors, which are the main component of LIB electrolytes, the direct detection of real LIB electrolytes was further investigated. As shown in Fig. 5a, the DNTT OFET sensor with biurea as the receptor layer showed a rapid response to $1 \mu \mathrm{L}$ electrolyte within $20 \mathrm{~s}$. Toward various volumes of LIB electrolyte $(1,5,10$, and $20 \mu \mathrm{L})$, the average responses of the
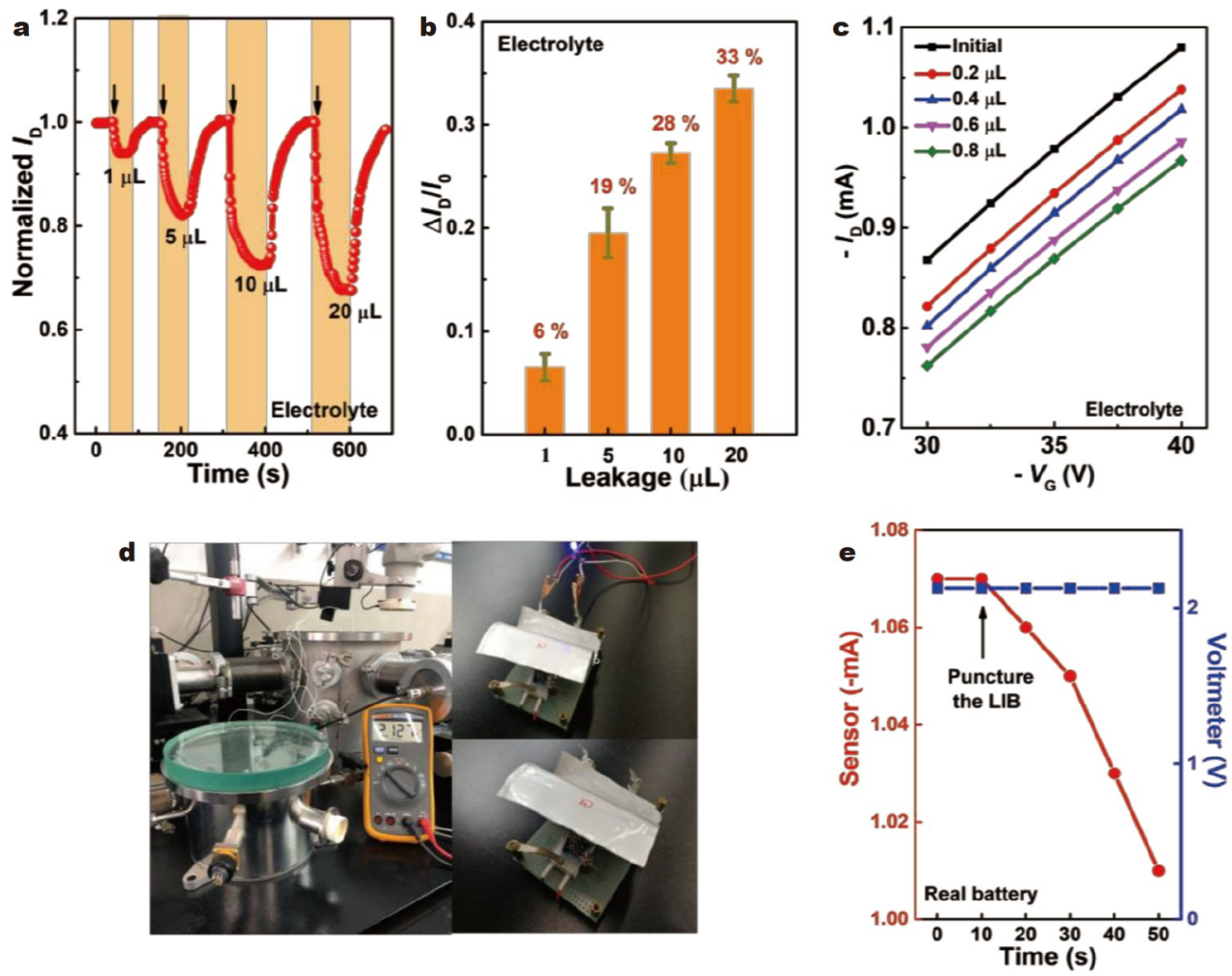

Figure 5 (a) The time-dependent responses of the DNTT OFET-based sensors with biurea receptor upon exposure to electrolyte at volumes ranging from 1 to $20 \mu \mathrm{L}$. $V_{\mathrm{G}}$ and $V_{\mathrm{D}}$ are fixed at -40 and $-10 \mathrm{~V}$, respectively. (b) Statistical comparison of the sensing results at volumes ranging from 1 to $20 \mu \mathrm{L}$. (c) Transfer curves of the DNTT OFET-based sensors with biurea receptor upon exposure to electrolyte at various volumes ranging from 0.2 to $0.8 \mu \mathrm{L}$. $V_{\mathrm{D}}=-10 \mathrm{~V}$. (d) Photographs of an LIB battery whose surface was pierced by a long metal needle and a real time monitor system to detect the leakage of LIB battery. (e) The time-dependent responses of the DNTT OFET-based sensors with biurea receptor (red line), and the real-time voltage monitoring by voltmeter (blue line) upon exposure to LIB electrolyte. 
sensor with narrow error bars were $6 \%, 19 \%, 28 \%$, and $33 \%$, respectively (Fig. 5b). The sensor could fully recover within a few seconds. As shown in Fig. 4b, there is a linear relationship between the current response and DEC concentration. From the relationship between the current response and the real LIB electrolyte (Fig. S18), the linear relationship is not good because the composition of the electrolyte is complicated and includes not only different organic solvents but also other additives. In addition, the OFET device has an upper concentration limit of over $200 \mathrm{~nL}$ electrolyte. The current of the device barely increases and is close to saturation, even with a higher volume of electrolyte injection. Moreover, the device exhibited effective detection of $0.2 \mu \mathrm{L}$ LIB electrolyte with a $3 \%$ current decrease and produced a higher response as the electrolyte volume increased from 0.2 to $0.8 \mu \mathrm{L}$ (Fig. $5 \mathrm{c}$ ). The excellent performance of the sensor was sufficient to meet the needs of detection of trace amounts of LIB electrolyte leakage.

To simulate real-time electrolyte leakage from a real LIB under working conditions, we assembled a set of real-time monitoring systems, including a homemade testing chamber, a voltmeter, a light-emitting diode (LED) bulb and a real LIB soft battery pack (Fig. 5d). The voltmeter and the LED bulb were connected to the battery pack over a period of time. Then, the surface of the battery was carefully pierced by a long metal needle. At the moment when the surface was punctured and thereafter, the reading of the voltmeter and the brightness of the LED barely changed. Although the LIB electrolyte continued to leak, the LED bulb remained on for a long time. This suggested that it was difficult to reveal whether there was any leakage of electrolyte by monitoring the output voltage of the LIBs. As a comparison, our sensor exhibited a significant response at the moment of leakage (Fig. 5e) and provided a timely warning. High-performance sensors are an important supplement to the current LIB safety monitoring system, and our sensors show great potential in applications in LIB electrolyte leakage monitoring.

\section{CONCLUSIONS}

In conclusion, for the first time, a highly sensitive and reliable OFET-based sensor to detect trace amounts of LIB electrolyte leakage was developed. Through a facile and convenient thermal evaporation process, $10-\mathrm{nm}$ OSC films of DNTT were prepared on silicon wafers, and on top of the OSC films, a 5-nm biurea film was then deposited as a receptor layer. Compared with the pristine DNTT OFET sensor, the receptor-decorated sensor exhibited much higher sensitivity, faster response and better recoverability. Due to the interactions between receptors and carbonate electrolyte vapors, the sensor showed a reliable and effective response to ultralow concentrations of DEC vapor (LOD of $1.4 \mathrm{ppm}$ ) or trace amounts of LIB electrolyte leakage $(200 \mathrm{~nL})$. In addition, the sensor showed excellent stability, and there was almost no change in performance over 500 days of testing in ambient atmosphere. More importantly, the receptordecorated DNTT OFET sensor could detect the leakage of electrolyte from an LIB in real time, while at the same time, the change in the voltage of the batteries with damaged surfaces was negligible, which showed the excellent early warning capabilities of our sensors. This work provides a low-cost, simple and feasible method that combines OFET-based sensors with specific receptors to improve their performance, realizes the rapid detection of redox neutral, flammable, explosive and volatile LIB electrolytes successfully, and may provide a new strategy for the design of OFET-based chemical sensors.

Received 21 October 2021; accepted 18 November 2021; published online 10 January 2022

1 Li M, Lu J, Chen Z, et al. 30 Years of lithium-ion batteries. Adv Mater, 2018, 30: 1800561

2 Wen J, Yu Y, Chen C. A review on lithium-ion batteries safety issues: Existing problems and possible solutions. Mat Express, 2012, 2: 197212

3 Golubkov AW, Fuchs D, Wagner J, et al. Thermal-runaway experiments on consumer Li-ion batteries with metal-oxide and olivin-type cathodes. RSC Adv, 2014, 4: 3633-3642

4 Li Y, Liu K, Foley AM, et al. Data-driven health estimation and lifetime prediction of lithium-ion batteries: A review. Renew Sustain Energy Rev, 2019, 113: 109254

5 Horsthemke F, Friesen A, Mönnighoff X, et al. Fast screening method to characterize lithium ion battery electrolytes by means of solid phase microextraction-gas chromatography-mass spectrometry. RSC Adv, 2017, 7: 46989-46998

6 Wilken S, Treskow M, Scheers J, et al. Initial stages of thermal decomposition of $\mathrm{LiPF}_{6}$-based lithium ion battery electrolytes by detailed Raman and NMR spectroscopy. RSC Adv, 2013, 3: 16359-16364

7 Di C, Zhang F, Zhu D. Multi-functional integration of organic fieldeffect transistors (OFETs): Advances and perspectives. Adv Mater, 2013, 25: 313-330

8 Guo Y, Yu G, Liu Y. Functional organic field-effect transistors. Adv Mater, 2010, 22: 4427-4447

9 Zhao G, Dong H, Jiang L, et al. Single crystal field-effect transistors containing a pentacene analogue and their application in ethanol vapor detection. Appl Phys Lett, 2012, 101: 103302

$10 \mathrm{Li} \mathrm{C}, \mathrm{Wu} \mathrm{H}$, Zhang $\mathrm{T}$, et al. Functionalized $\pi$ stacks of hexabenzoperylenes as a platform for chemical and biological sensing. Chem, 2018, 4: 1416-1426

11 Li H, Dailey J, Kale T, et al. Sensitive and selective $\mathrm{NO}_{2}$ sensing based on alkyl- and alkylthio-thiophene polymer conductance and conductance ratio changes from differential chemical doping. ACS Appl Mater Interfaces, 2017, 9: 20501-20507

12 Lu Y, Zhang S, Dai S, et al. Ultrasensitive detection of electrolyte leakage from lithium-ion batteries by ionically conductive metalorganic frameworks. Matter, 2020, 3: 904-919

13 Zhang F, Qu G, Mohammadi E, et al. Solution-processed nanoporous organic semiconductor thin films: Toward health and environmental monitoring of volatile markers. Adv Funct Mater, 2017, 27: 1701117

14 Yuan Y, Giri G, Ayzner AL, et al. Ultra-high mobility transparent organic thin film transistors grown by an off-centre spin-coating method. Nat Commun, 2014, 5: 3005

15 Zhang C, Chen P, Hu W. Organic field-effect transistor-based gas sensors. Chem Soc Rev, 2015, 44: 2087-2107

16 Zhang S, Zhao Y, Du X, et al. Gas sensors based on nano/microstructured organic field-effect transistors. Small, 2019, 15: 1805196

17 Ji $\mathrm{D}, \mathrm{Li} \mathrm{T}, \mathrm{Hu} \mathrm{W}$, et al. Recent progress in aromatic polyimide dielectrics for organic electronic devices and circuits. Adv Mater, 2019, 31: 1806070

18 Ji S, Wang $\mathrm{H}$, Wang $\mathrm{T}$, et al. A high-performance room-temperature $\mathrm{NO}_{2}$ sensor based on an ultrathin heterojunction film. Adv Mater, 2013, 25: $1755-1760$

19 Lu J, Liu D, Zhou J, et al. Porous organic field-effect transistors for enhanced chemical sensing performances. Adv Funct Mater, 2017, 27: 1700018

20 Wu X, Mao S, Chen J, et al. Strategies for improving the performance of sensors based on organic field-effect transistors. Adv Mater, 2018, 30: 1705642

21 Zang Y, Huang D, Di CA, et al. Device engineered organic transistors for flexible sensing applications. Adv Mater, 2016, 28: 4549-4555

22 Khim D, Ryu GS, Park WT, et al. Precisely controlled ultrathin conjugated polymer films for large area transparent transistors and highly sensitive chemical sensors. Adv Mater, 2016, 28: 2752-2759

23 Li L, Gao P, Baumgarten M, et al. High performance field-effect am- 
monia sensors based on a structured ultrathin organic semiconductor film. Adv Mater, 2013, 25: 3419-3425

24 Wang $\mathrm{Z}$, Huang $\mathrm{L}$, Zhu $\mathrm{X}$, et al. An ultrasensitive organic semiconductor $\mathrm{NO}_{2}$ sensor based on crystalline TIPS-pentacene films. Adv Mater, 2017, 29: 1703192

25 Zhang F, Di C, Berdunov N, et al. Ultrathin film organic transistors: Precise control of semiconductor thickness via spin-coating. Adv Mater, 2013, 25: 1401-1407

26 Lee MY, Kim HJ, Jung GY, et al. Highly sensitive and selective liquidphase sensors based on a solvent-resistant organic-transistor platform. Adv Mater, 2015, 27: 1540-1546

27 Shen H, Zou Y, Zang Y, et al. Molecular antenna tailored organic thinfilm transistors for sensing application. Mater Horiz, 2018, 5: 240-247

28 Sokolov AN, Roberts ME, Johnson OB, et al. Induced sensitivity and selectivity in thin-film transistor sensors via calixarene layers. Adv Mater, 2010, 22: 2349-2353

29 Torsi L, Farinola GM, Marinelli F, et al. A sensitivity-enhanced fieldeffect chiral sensor. Nat Mater, 2008, 7: 412-417

30 Li H, Shi Y, Han G, et al. Monolayer two-dimensional molecular crystals for an ultrasensitive OFET-based chemical sensor. Angew Chem Int Ed, 2020, 59: 4380-4384

31 Lin P, Yan F. Organic thin-film transistors for chemical and biological sensing. Adv Mater, 2012, 24: 34-51

32 Meng Q, Zhang F, Zang Y, et al. Solution-sheared ultrathin films for highly-sensitive ammonia detection using organic thin-film transistors. J Mater Chem C, 2014, 2: 1264-1269

33 Peng B, Huang S, Zhou Z, et al. Solution-processed monolayer organic crystals for high-performance field-effect transistors and ultrasensitive gas sensors. Adv Funct Mater, 2017, 27: 1700999

34 Jang M, Kim H, Lee S, et al. Highly sensitive and selective biosensors based on organic transistors functionalized with cucurbit[6]uril derivatives. Adv Funct Mater, 2015, 25: 4882-4888

35 Jang $\mathrm{Y}$, Jang $\mathrm{M}$, Kim $\mathrm{H}$, et al. Point-of-use detection of amphetaminetype stimulants with host-molecule-functionalized organic transistors. Chem, 2017, 3: 641-651

36 Witte J, Neaton JB, Head-Gordon M. Effective empirical corrections for basis set superposition error in the def2-SVPD basis: gCP and DFT-C. J Chem Phys, 2017, 146: 234105

37 Tan $\mathrm{H}$, Chen $\mathrm{Q}$, Chen $\mathrm{T}$, et al. Selective adsorption and separation of xylene isomers and benzene/cyclohexane with microporous organic polymers POP-1. ACS Appl Mater Interfaces, 2018, 10: 32717-32725

38 Lu T, Chen F. Multiwfn: A multifunctional wavefunction analyzer. J Comput Chem, 2012, 33: 580-592

Acknowledgements This work was supported by the National Natural Science Foundation of China (61822405 and 62074111), the Science \& Technology Foundation of Shanghai (19JC1412402 and 20JC1415600), Shanghai Municipal Science and Technology Major Project (2021SHZDZX0100), Shanghai Municipal Commission of Science and Technology Project (19511132101), and the Fundamental Research Funds for the Central Universities. We also acknowledge the help from Dr. Howard E. Katz on discussion about the selection of the receptors.

Author contributions Zhang S performed the most tests and wrote the manuscript; Lu Y, Li L, Wang X, Zhang J, Sun Q and Huang Y helped with tests and characterization analysis; Liu D and Dai S helped build the test system; Hao D and Yang B helped analyze the data; Wei L provided theoretical calculations; Huang Y supervised the battery leakage test; Huang J developed the concept and provided financial support through grant applications. All authors contributed to the general discussion.

Conflict of interest The authors declare that they have no conflict of interest.

Supplementary information Supporting data are available in the online version of the paper.

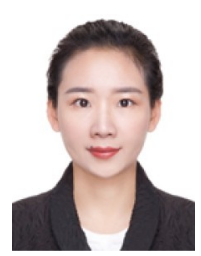

Shiqi Zhang received her Bachelor's degree from the College of Nano Science and Technology, Soochow University. Now, she is pursuing her $\mathrm{PhD}$ degree under the supervision of Prof. Jia Huang at Tongji University. Her current research interest is organic thin-film transistors and chemical sensors.

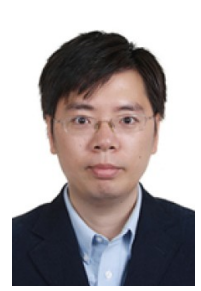

Jia Huang is a professor of materials science and engineering at Tongji University, Shanghai, China. He received his BSc degree in materials science and engineering from the University of Science and Technology of China, Hefei, China, MSc degree in applied science from the College of William \& Mary, Williamsburg, VA, USA, and $\mathrm{PhD}$ degree in materials science and engineering from Johns Hopkins University, Baltimore, MD, USA. His research focuses on chemical sensors and thin-film transistors.

\section{基于受体修饰的双层有机场效应晶体管化学传感器 用于高灵敏检测锂离子电池电解液泄漏}

张诗琦 $^{1}$, 陆洋 ${ }^{1}$, 李立 ${ }^{1}$, 王秷 ${ }^{1}$, 刘 鹏 $^{1}$, 张钧尧 ${ }^{1}$, 代世磊 ${ }^{1}$, 郝丹丹 ${ }^{1}$, 杨奔 ${ }^{1}$, 孙全 $^{2}$, 黄云辉 ${ }^{2}$, 魏莱 $^{3}$, 黄佳 ${ }^{1,4^{*}}$

摘要 易燃易爆的锂离子电池电解液泄漏是电池故障的早期症状之一, 甚至会导致电池自燃或电动汽车爆炸. 因此, 有必要找到一种快速简单 的方法来监测电解液泄漏. 现有的传感器难以有效且快速地检测微量 电解液泄漏. 我们首次提出了一种将有机场效应晶体管(OFET)的灵敏 度和联二艮受体的选择性相结合, 以检测电池电解液泄漏的策略. 复合 后的传感器与没有受体的原始传感器相比, 灵敏度显著提高, 传感器对 碳酸二乙酯的检测限低至 $1.4 \mathrm{ppm}$. 并且可以在几秒钟内有效地检测到 微量的泄漏, $200 \mathrm{~nL}$ 电解液泄漏能够导致 $3 \%$ 的响应. 受体复合后的 OFET传感器的优异性能使其成为锂离子电池安全监测的良好候选者, 并为传感技术的发展提供了一个有前途的平台. 